\title{
A flagellin-adjuvanted inactivated porcine epidemic diarrhea virus (PEDV) vaccine provides enhanced immune protection against PEDV challenge in piglets
}

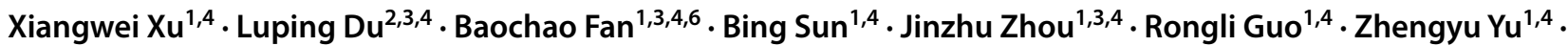 \\ Danyi Shi ${ }^{1,5} \cdot$ Kongwang $\mathrm{He}^{1,3,4} \cdot{\mathrm{Bin} \mathrm{Li}^{1,3,4,6}}^{1,4}$
}

Received: 15 May 2019 / Accepted: 28 January 2020 / Published online: 6 April 2020

(c) Springer-Verlag GmbH Austria, part of Springer Nature 2020

\begin{abstract}
Since late 2010, outbreaks of porcine epidemic diarrhea (PED) have been reported in the swine industry in China. A variant PEDV strain that differs from strain CV777 causes prevalent PEDV infections which commercial vaccines based on CV777 cannot provide complete protection. In this study, we designed a new vaccine based on the epidemic PEDV strain AH2012/12, adjuvanted with flagellin, a mucosal adjuvant that induces mucosal and systemic production of IgA. Three groups of pregnant sows were immunized twice, with a 14-day interval, with PEDV adjuvanted with flagellin, PEDV alone, or PBS before farrowing, and newborn piglets from each group were selected and challenged with PEDV. Immunization with this vaccine elicited high levels of IgG, IgA, and neutralizing antibodies in the serum and colostrum of sows, and newborn piglets were protected against PEDV while suckling. This study should guide the prevention and control strategies for PEDV infection, thereby reducing the losses associated with this virus.
\end{abstract}

\section{Introduction}

Porcine epidemic diarrhea virus (PEDV), the etiological agent of porcine epidemic diarrhea (PED), causes acute watery diarrhea, dehydration, vomiting, and high mortality

Handling Editor: Diego G. Diel.

Xiangwei Xu and Luping Du contributed equally to this work.

Kongwang $\mathrm{He}$

Kongwanghe@126.com

$凶 \operatorname{Bin} \mathrm{Li}$

libinana@126.com

1 Key Laboratory of Veterinary Biological Engineering and Technology, Ministry of Agriculture, Institute of Veterinary Medicine, Jiangsu Academy of Agricultural Sciences, 50 Zhong-ling Street, Nanjing 210014, Jiangsu, People's Republic of China

2 Institute of Veterinary Immunology and Engineering, Jiangsu Academy of Agricultural Sciences, Nanjing 210014, Jiangsu, People's Republic of China

3 Jiangsu Co-Innovation Center for the Prevention and Control of Important Animal Infectious Disease and Zoonose, Yangzhou University, Yangzhou 225009, People's Republic of China in neonatal piglets. At the end of 2010, a major increase in PED outbreaks occurred in the pig-producing provinces of China, despite continued use of the available vaccines based on the CV777 strain of PEDV. Inactivated or attenuated vaccines for PEDV (strain CV777, subgroup GI-a) and transmissible gastroenteritis virus (TGEV) were approved in China in 1995 and 1998, respectively. In 2015, a trivalent vaccine consisting of attenuated PEDV (strain CV777,

4 Jiangsu Key Laboratory for Food Quality and Safety-State Key Laboratory Cultivation Base of Ministry of Science and Technology, Nanjing 210014, Jiangsu, People's Republic of China

5 College of Animal Science and Technology, Hebei Normal University of Science and Technology, Qinhuangdao, People's Republic of China

6 School of Food and Biological Engineering, Jiangsu University, Zhenjiang 212013, People's Republic of China 
subgroup GI-a), TGEV, and porcine rubulavirus (PoRV) and a dual attenuated vaccine combining TGEV and PEDV (strain ZJ08, subgroup GI-b) were officially launched on the market [28]. Recent epidemics may be attributable to variants of PEDV [13, 22]. In a previous study, an epidemic PEDV field strain, AH2012/12 (accession no. KU646831), from a pig farm in Anhui Province reporting severe diarrhea was isolated in our laboratory and serially propagated in cell culture for over 50 passages [12]. This strain is most closely related to emergent PEDV strains in America [12] and is reported to be highly pathogenic in newborn piglets [12]. Therefore, we prepared an inactivated PEDV vaccine based on strain AH2012/12 to develop an effective preventive and control measure against PEDV.

Considering the route of PEDV infection, a mucosal adjuvant, flagellin, was used in the development of the inactivated PEDV vaccine (Vac201-FliC), prepared by mixing the inactivated PEDV vaccine antigens with Montanide ${ }^{\mathrm{TM}}$ ISA201 adjuvant and then adding flagellin (FliC) before use. Flagellin is a Toll-like receptor 5 (TLR5) ligand, suggesting its potential utility as an adjuvant [20]. Unlike many TLR agonists, flagellin tends to produce mixed Th1 and Th2 cell responses rather than a strongly polarized Th1 response [16]. It has been reported that the mucosal administration of flagellin increases the levels of mucosal and systemic immunoglobulin (IgA) [14, 18].

In this study, we evaluated the immune responses of pregnant sows and suckling piglets induced by the administration of phosphate-buffered saline (PBS), Vac201 (inactivated PEDV mixed with ISA201), or Vac201-FliC (a mix composed of inactivated PEDV, ISA201 and FliC). The results indicated that Vac201-FliC induced significantly more potent immune responses, reflected by serum $\operatorname{IgG}$ and $\operatorname{IgA}$ antibody titers and colostrum IgA antibody titers in pregnant sows, than PBS or Vac201, and it efficiently protected suckling piglets from challenge with PEDV.

\section{Materials and methods}

\section{Cells and viruses}

The Vero-81 (ATCC CCL-81) cell line was used for propagation of PEDV. Vero cells were maintained in Dulbecco's modified Eagle's medium (DMEM; Life Technologies, Carlsbad, CA, USA) supplemented with 5\% (v/v) heatinactivated fetal bovine serum (FBS; Life Technologies), penicillin (100 units/mL), streptomycin $(100 \mathrm{mg} / \mathrm{mL})$, and Fungizone $(0.25 \mathrm{mg} / \mathrm{mL})$ (Life Technologies). PEDV strain AH2012/12 (GenBank accession no. KU646831) was isolated and maintained in our laboratory as described previously $[9,17,29]$. As reported, AH2012/12 was genetically distinct from the vaccine strains CV777 and attenuated
DR-13, with nucleotide sequence identity ranging from $96.7 \%$ to $96.8 \%$.

\section{Cloning and expression of flagellin (FliC)}

The flagellin gene (FliC) was amplified from swine Salmonella using the pair of specific primers listed in Table 1 (GenBank accession no. CP011259.2). The PCR product was cloned into the prokaryotic expression vector pET28a between the BamHI and HindIII sites. The ligated product was initially propagated in competent Escherichia coli cells (Takara, Dalian, China). The transformed colonies were screened by restriction enzyme digestion and DNA sequencing. The recombinant pET28a-FliC plasmid was extracted from the $E$. coli cells, purified, and used to transform $E$. coli BL21 (DE3) cells (Takara, Dalian, China) for flagellin expression. FliC expression was induced by the addition of $1 \mathrm{mM}$ isopropy1- $\beta$-D-1-thiogalactopyranoside (IPTG) (Zhuyan, Nanjing, China) to the transformed BL21 (DE3) bacteria when they reached an optical density at $600 \mathrm{~nm}$ $\left(\mathrm{OD}_{600}\right)$ of 0.6 at $37^{\circ} \mathrm{C}$. The samples were collected after $6 \mathrm{~h}$ and analyzed by sodium dodecyl sulfate polyacrylamide gel electrophoresis (SDS-PAGE). The recombinant protein was confirmed by western blotting analysis using an anti-His-tag monoclonal antibody (Boster, Wuhan, China).

The FliC proteins were purified using Ni-NTA spin columns (QIAGEN, Hilden, Germany) under denaturing conditions as per the manufacturer's instructions. Purified protein was quantitated using the Bradford assay and stored in the same buffer at $-20{ }^{\circ} \mathrm{C}$ until use. Endotoxins were removed using ToxinEraser ${ }^{\mathrm{TM}}$ endotoxin removal resin from GenScript.

\section{Preparation of vaccine antigens and two formulations of inactivated PEDV vaccine}

A Vero-81 cell monolayer infected with PEDV strain AH2012/12 (inoculated at a multiplicity of infection of 0.05) was maintained in DMEM containing $10 \mu \mathrm{g}$ of trypsin per $\mathrm{mL}$ at $37{ }^{\circ} \mathrm{C}$ in a $5 \% \mathrm{CO}_{2}$ atmosphere until a cytopathic effect was apparent. The infected cells were lysed by the freeze-thaw method and centrifuged at $2,000 \times g$ for $10 \mathrm{~min}$.

Table 1 Primer sequence for amplification of porcine FliC.

\begin{tabular}{lll}
\hline Name & Sequence & Amplified gene \\
\hline FliC-F & GAATTCGGATCCATGGCA & FliC \\
& CAAGTCATTAAT & \\
& BamH I & \\
FliC-R & CTCGAGAAGCTTACGCAG & \\
& TAAAGAGAG & \\
& Hind III & \\
\hline
\end{tabular}


Virions in the supernatant were quantitated and adjusted to $10^{7}$ median tissue culture infective doses $\left(\mathrm{TCID}_{50}\right) / \mathrm{mL}$. The inactivated vaccines were prepared by treating the virus with $0.1 \%$ formaldehyde at $37^{\circ} \mathrm{C}$ for $48 \mathrm{~h}$. The killed virus was stored at $-70{ }^{\circ} \mathrm{C}$.

The Montanide ${ }^{\mathrm{TM}}$-ISA201-emulsified killed PEDV vaccine (Vac201) was prepared by mixing the inactivated PEDV vaccine antigens $\left(10^{6} \mathrm{TCID}_{50}\right)$ in an equal volume of Montanide ${ }^{\mathrm{TM}}$ ISA201 adjuvant (SEPPIC, Shanghai, China) as instructed by the manufacturer. The Montanide ${ }^{\mathrm{TM}}$ ISA201-emulsified killed PEDV vaccine plus flagellin protein (Vac201-FliC) was prepared by mixing the inactivated PEDV vaccine antigens $\left(10^{6} \mathrm{TCID}_{50}\right)$ in an equal volume of Montanide ${ }^{\mathrm{TM}}$ ISA201 adjuvant and then adding flagellin $(100 \mu \mathrm{g})$ before use.

\section{Pigs and inoculation}

Nine healthy commercial sows (Large White, Primi-para) from a PEDV-negative farm were selected and confirmed to be negative for PEDV, TGEV and porcine deltacoronavirus by reverse transcription (RT)-PCR. The animals were confirmed to be serologically negative for PEDV and TGEV antibodies using an indirect enzyme-linked immunosorbent assay (ELISA). They were then randomly divided into three groups (three sows per group), which were housed in three separate rooms.

As shown in Fig. 1 , the three groups were immunized intranasally with $4 \mathrm{~mL}$ of PBS, Vac201, or Vac201-FliC at 28 and 14 days before farrowing. Each dose of the PEDV vaccine (Vac201 or Vac201-FliC) was equivalent to approximately $10^{6} \mathrm{TCID}_{50}$ of inactivated virus. Serum samples were collected 28 and 14 days before farrowing, and colostrum was collected after delivery for the detection of N-proteinspecific antibodies and PEDV-neutralizing antibodies according to a previously published method $[10,26]$. The colostrum samples were also tested for the presence of IgA antibodies directed against PEDV, using commercial ELISA kits (AniGen PED IgA Ab ELISA) (Antigen, Korea). When the pregnant sows were farrowing, fresh peripheral blood mononuclear cells (PBMCs) were collected for lymphocyte proliferation and interferon (IFN)- $\gamma$ release assays.
After farrowing, the litter sizes of surviving newborn piglets from one sow ranged from 7 to 18 . After birth, newborn piglets were fed with the sows' colostrum. At 5 days post-farrowing, five piglets $(1.35-1.55 \mathrm{~kg})$ were randomly selected from each sow in a treatment group. Numbered piglets were housed in isolation rooms and artificially fed with milk. Then, the piglets were orally challenged with 100 median lethal doses $\left(\mathrm{LD}_{50}\right)$ of PEDV AH2012/12 (passage $10,10^{7} \mathrm{TCID}_{50}, 2 \mathrm{ml}$ ). Prior to challenge, sera were collected from the piglets for the detection of PEDV-Nprotein-specific IgG antibodies and neutralizing antibodies. The challenged piglets were monitored at $0,6,12,24,48$, 72,96 and $120 \mathrm{~h}$ post-challenge for clinical signs of diarrhea or death.

\section{ELISA}

Titers of PEDV-N-protein-specific antibodies in the serum and colostrum were determined using an endpoint ELISA with purified PEDV N protein as the antigen as described previously with some modifications [19, 26]. Briefly, ELISA plates were coated with purified PEDV N protein $(0.8 \mu \mathrm{g} /$ $\mathrm{mL})$ in carbonate-bicarbonate buffer $(\mathrm{pH} 9.6)$ and then washed and blocked with $5 \%$ skimmed milk $+0.1 \%$ Tween 20 in PBS. Twofold serially diluted serum samples (starting with a dilution of 1:8) were added and incubated for $1 \mathrm{~h}$ at $37{ }^{\circ} \mathrm{C}$. PEDV-N-protein-specific antibody was detected using anti-pig IgG secondary antibodies conjugated to horseradish peroxidase (HRP) (KPL). Plates were developed using 3,3',5,5'-tetramethylbenzidine, and absorbance was measured at $450 \mathrm{~nm}$. The titers were expressed as the reciprocal of the highest dilution of serum producing a signal that was more than twice the background level.

PEDV-specific IgA antibodies in the colostrum were analyzed using an AniGen PED IgA Ab ELISA kit (Anigen, Korea) as instructed by the manufacturer.

\section{Serum neutralization test}

The serum neutralization test was performed according to a previously published method [26], with some modifications. Swine serum and colostrum samples were inactivated at $56{ }^{\circ} \mathrm{C}$ for $30 \mathrm{~min}$ and stored at $-20{ }^{\circ} \mathrm{C}$ until use. After

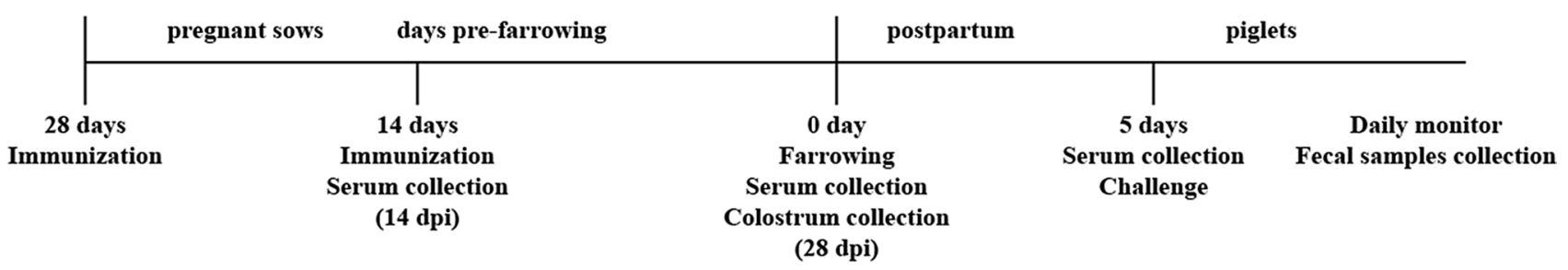

Fig. 1 Experimental design 
twofold dilution, serum or colostrum was mixed with PEDV (200 TCID $50 / 0.1 \mathrm{~mL}$ ) in equal volumes, and incubated for $1 \mathrm{~h}$ at $37^{\circ} \mathrm{C}$. Subsequently, $0.1 \mathrm{~mL}$ of each mixture was transferred to Vero cell monolayers in a 96-well tissue culture plate and washed twice with PBS. After adsorption for $1 \mathrm{~h}$ at $37{ }^{\circ} \mathrm{C}$, inocula were discarded, and the cells were washed twice with PBS. Next, maintenance medium containing trypsin $(10 \mu \mathrm{g} / \mathrm{mL})$ was added to each well, and the plate was incubated for 5 days at $37^{\circ} \mathrm{C}$. The neutralization titers were expressed as the reciprocal of the highest serum or colostrum dilution resulting in complete neutralization. Each sample was assayed in triplicate.

\section{Lymphocyte proliferation assay}

Lymphocyte proliferation assays were performed using PBMCs from pregnant sows at farrowing, obtained by density centrifugation. The PBMCs were collected and stimulated with or without PEDV proteins $(20 \mu \mathrm{g} / \mathrm{mL})$, which were prepared by ultracentrifugation of Vero-81 cells infected with PEDV. Lymphocyte proliferation assays were performed as described previously [11]. T-cell stimulation was expressed as the stimulation index (SI), which was calculated as the ratio of the average OD values of the antigenstimulated wells to those of the unstimulated wells.

\section{IFN- $\gamma$ release assay}

To assay PEDV-specific IFN- $\gamma$-secreting PBMCs isolated from pigs immunized with Vac201-FliC, Vac201 or PBS, isolated PBMCs $\left(1 \times 10^{6}\right.$ cells $\left./ \mathrm{mL}\right)$ were cultured in 24-well plates at $37{ }^{\circ} \mathrm{C}$ in the presence of $5 \% \mathrm{CO}_{2}$ and stimulated with PEDV proteins at a concentration of $20 \mu \mathrm{g} / \mathrm{mL}$. After incubation for $72 \mathrm{~h}$, the cells were centrifuged and the culture supernatant was harvested. IFN- $\gamma$ was quantitated using a commercial porcine IFN- $\gamma$ ELISA kit (ELISA kit for Interferon Gamma (IFNg, USCN, Wuhan, China) according to the manufacturer's protocol. The concentration of IFN- $\gamma$ in the samples was determined from a standard curve.

\section{Real-time PCR analysis of IFN- $\gamma$ mRNA expression}

PBMCs were cultured in 24-well plates at $1 \times 10^{6}$ cells $/ \mathrm{mL}$ for $18 \mathrm{~h}$ at $37^{\circ} \mathrm{C}$ with $5 \% \mathrm{CO}_{2}$ in the presence or absence of $20 \mu \mathrm{g}$ of PEDV proteins per mL. Real-time PCR was performed as described previously [19]. Relative IFN- $\gamma$ gene expression was analyzed as described previously [11], and IFN- $\gamma$ gene expression was normalized to GAPDH expression. The relative difference in IFN- $\gamma$ expression among different groups was determined as $2^{-\Delta \Delta \mathrm{Ct}}$. The genespecific primers used were also described previously [11]: pig- IFN- $\gamma$, 5'-AGAATTGGAAAGAGGAGAGTGACAA/ TGAATGGCCTGGTTATCTTTGA-3'; pig-glyceraldehyde 3-phosphate dehydrogenase (GAPDH), 5'-ACATGGCCT CCA AGGAGTAAGA/GATCGAGTTGGGGCTGTG ACT-3'.

\section{Clinical evaluation}

All piglets were monitored daily after challenge for clinical signs of disease, including diarrhea and vomiting. Rectal swabs were collected to score fecal consistency (scores: 0 , normal; 1, pasty stool; 2, semiliquid diarrhea; and 3, liquid diarrhea) [12, 19]. Fecal PEDV shedding post-challenge was tested by RT-quantitative PCR (RT-qPCR). The primers and probes targeting conserved regions in the PEDV N protein gene have been described previously [12]. The intestinal tissues were grossly evaluated at necropsy. Fresh jejuna and ilea were collected and fixed in 10\% neutral-buffered formalin for histopathological examination.

\section{Statistical analysis}

Statistical analysis was performed using GraphPad Prism version 5 (GraphPad Software, San Diego, CA, USA) and IBM SPSS Statistic 20 . The data were analyzed by one-way analysis of variance, followed by Tukey's $t$-test or Student's $t$-test. The survival curve shown in Figure 7C was analyzed by Kaplan-Meier survival curve analysis. A $P$-value less than 0.05 indicated a statistically significant difference. All data are expressed as the mean \pm standard error of the mean (SEM).

\section{Ethics approval}

The study and study protocol were approved by the Science and Technology Agency of Jiangsu Province. Approval was also granted by the Jiangsu Academy of Agricultural Sciences Experimental Animal Ethics Committee (approval ID NKYVET 2015-2016). All efforts were made to minimize the animals' suffering. The immunization, challenge, and collection of serum and colostrum samples were performed in strict accordance with the guidelines of the Jiangsu Province Animal Regulations (Government Decree no. 45).

\section{Results}

\section{Expression and purification of FliC}

A single PCR product with an estimated length of $1503 \mathrm{bp}$ (the length of FliC) was amplified from swine Salmonella (Fig. 2A). The fragment was then cloned in-frame into the pET28a vector, and successful insertion was confirmed by double digestion with BamHI and HindIII (Fig. 2B). The 
Fig. 2 A. Subcloning the FliC gene. Lanes 1-2: PCR products of FliC; lane M, DL2000 DNA Marker (Takara, Dalian, China). B. pET28a-FliC digested with BamHI and HindIII. Lane 1, pET28a-FliC; lane M, marker DL2000. C. Expression of flagellin. Lanes 1-3: SDS-PAGE analysis of flagellin. Lane 1, pET28a; lane 2, pET28a-FliC; lane 3 , purified flagellin. D. Western blotting analysis of flagellin with an anti-His tag monoclonal antibody. Lane 1 , pET28a; lane 2, pET28a-FliC
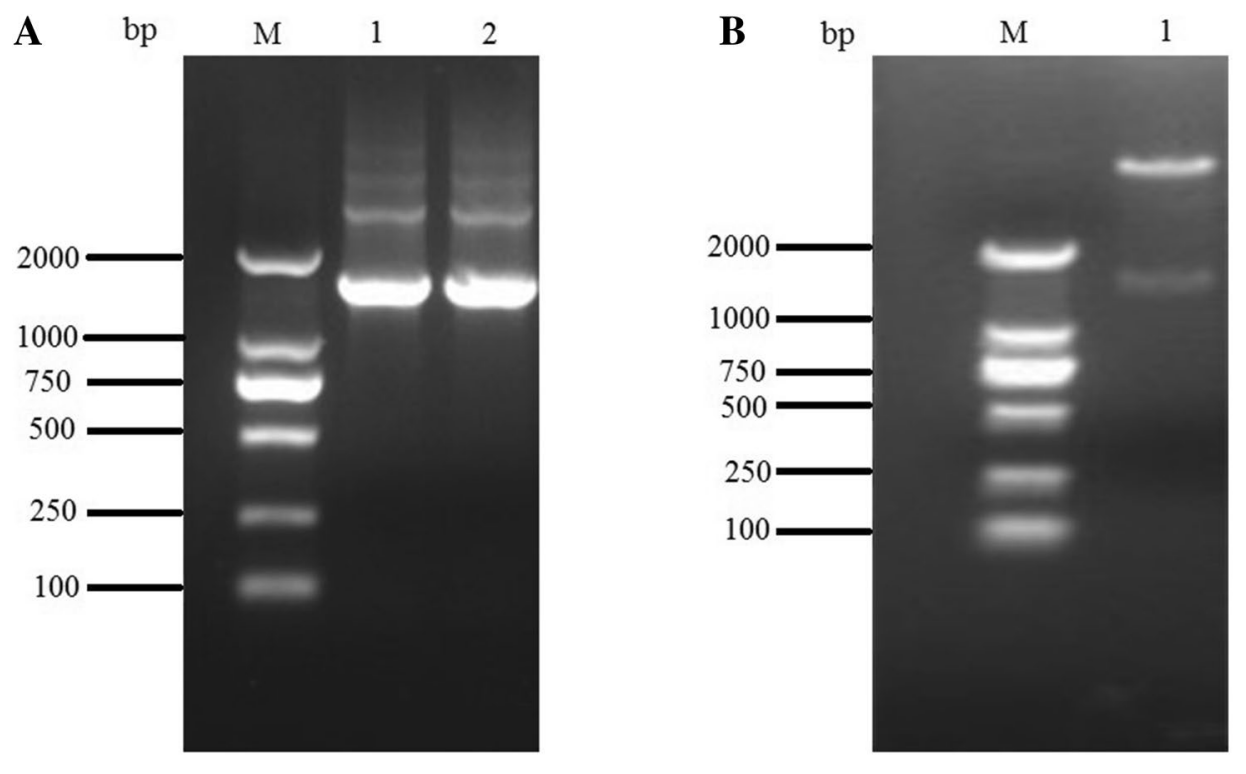

C

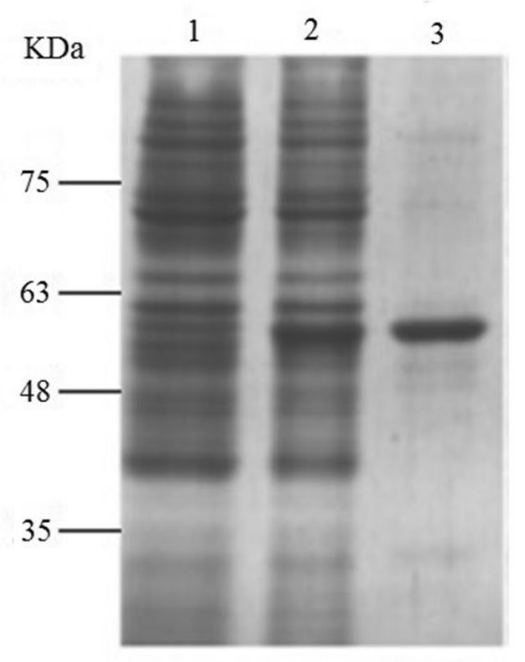

D

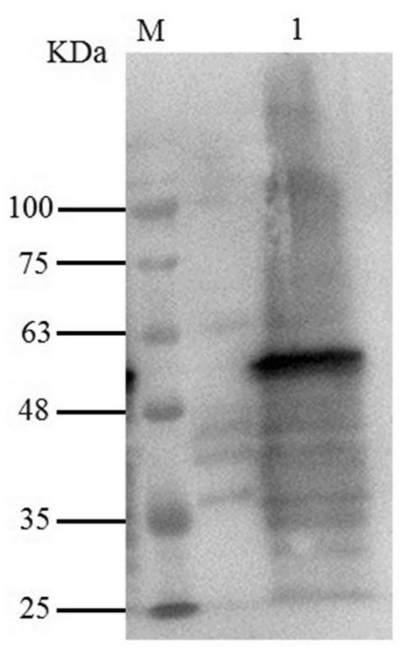

purified FliC protein was analyzed by SDS-PAGE (Fig. 2C) and Western blotting (Fig. 2D).

\section{Antibody responses in pregnant sows}

Antibody responses induced by Vac201-FliC in pregnant sows are one of the most important correlates of immunity against PEDV infection in piglets. Therefore, we analyzed antibody responses in immunized pregnant sows using an indirect ELISA. As shown in Fig. 3A, Vac201-FliC induced the highest PEDV-N-protein-specific IgG antibody titers in the serum at both 14 and 28 days post-immunization (dpi). At 14 and 28 dpi, the titers of PEDV-N-protein-specific IgG antibodies were numerically higher in animals immunized with Vac201-FliC than in animals immunized with
Vac201, but these differences were not statistically significant $(P>0.05)$.

The neutralization potency of sera from the immunized pigs was also investigated. As shown in Fig. 3B, Vac201FliC induced the highest titers of serum neutralizing antibodies at 14 and 28 dpi. Vac201 and Vac201-FliC induced significantly higher titers of serum neutralizing antibodies than PBS alone $(P<0.05)$. Furthermore, at 14 and $28 \mathrm{dpi}$, the PEDV-specific IgA levels in the serum samples were significantly higher in animals vaccinated with Vac201-FliC than in animals vaccinated with PBS or Vac201 $(P<0.01$ and $P<0.001$, respectively).

In swine, colostrum is one of the most important sources of PEDV-specific IgG antibody transferred to piglets to prevent PEDV infection. The PEDV-N-protein-specific antibody titers and neutralizing antibody titers in the colostrum 
Fig. 3 Serum antibody responses in vaccinated pregnant sows. Serum samples were collected at 14 and 28 days after the primary immunization to determine the levels of PEDV-N-protein-specific IgG antibodies (A), neutralizing antibodies against PEDV strain AH2012/12 (B), and PEDV-specific IgA antibodies (C). Data are presented as the mean \pm SEM of three sows per group
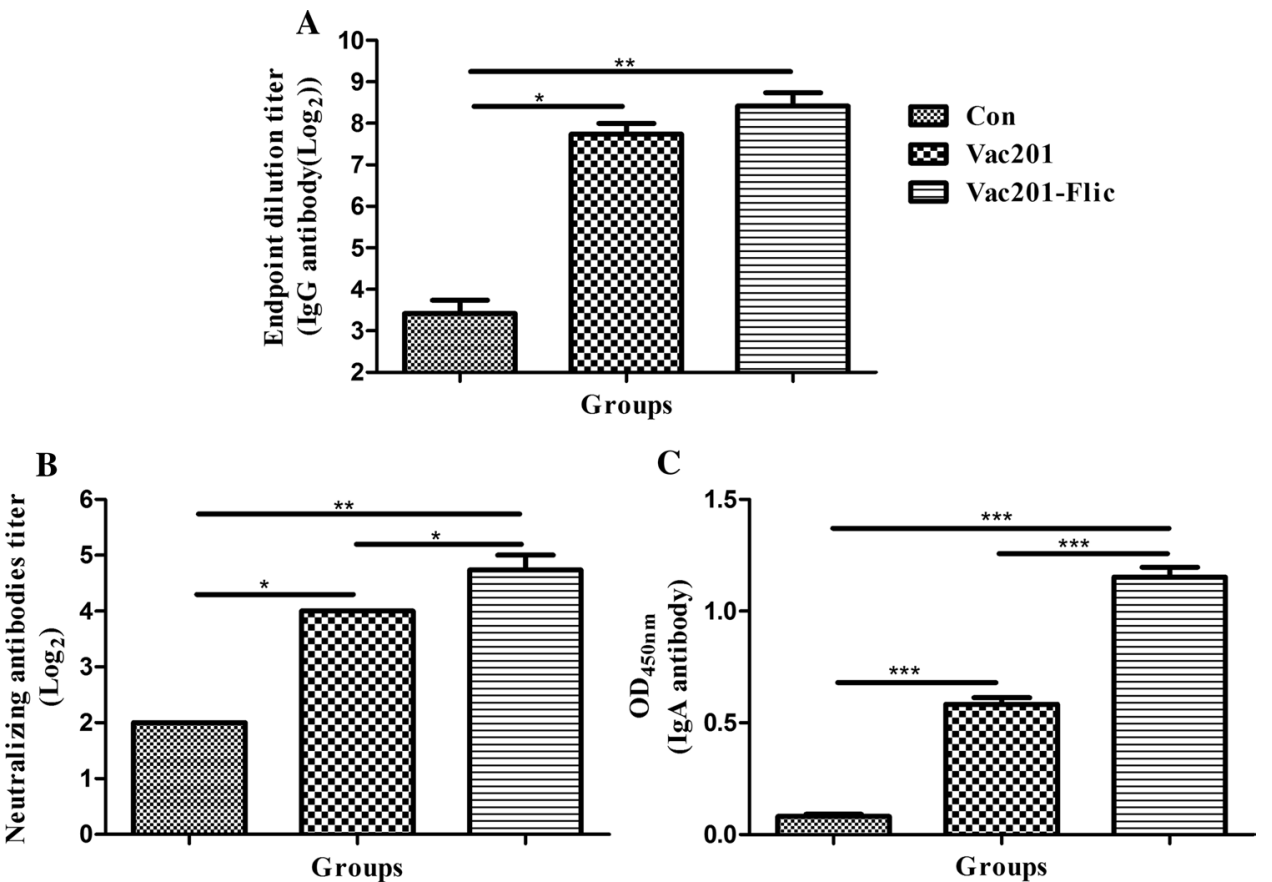

samples were also determined to evaluate the efficacy of Vac201-FliC. The PEDV-N-protein-specific antibody titers in the colostrum samples from animals vaccinated with Vac201-FliC differed significantly from the serum titers of animals immunized with PBS alone $(P<0.01)$. The titer of $\mathrm{N}$-protein-specific antibodies was higher in the colostrum samples from the animals immunized with Vac201-FliC than in those from the animals immunized with Vac201, but this difference was not statistically significant $(P>0.05)$. As shown in Fig. 4B, significant differences in the levels of neutralizing antibodies in the colostrum samples were observed between the Vac201-FliC-vaccinated and PBS-vaccinated groups $(P<0.01)$, and between the Vac201-FliC-vaccinated and Vac201-vaccinated groups $(P<0.05)$.

The ability of FliC to induce a mucosal immune response was also analyzed by determining the PEDVspecific IgA antibody titers induced by Vac201-FliC in the pregnant sows. Because IgA levels correlate directly
Fig. 4 Colostral antibodies from vaccinated pregnant sows. Colostrum samples were collected after delivery to determine the colostral levels of PEDV-N-protein-specific IgG antibodies (A), neutralizing antibodies against PEDV strain AH2012/12 (B), and PEDV-specific IgA antibodies (C). Data presented are the mean \pm SEM of three sows per group
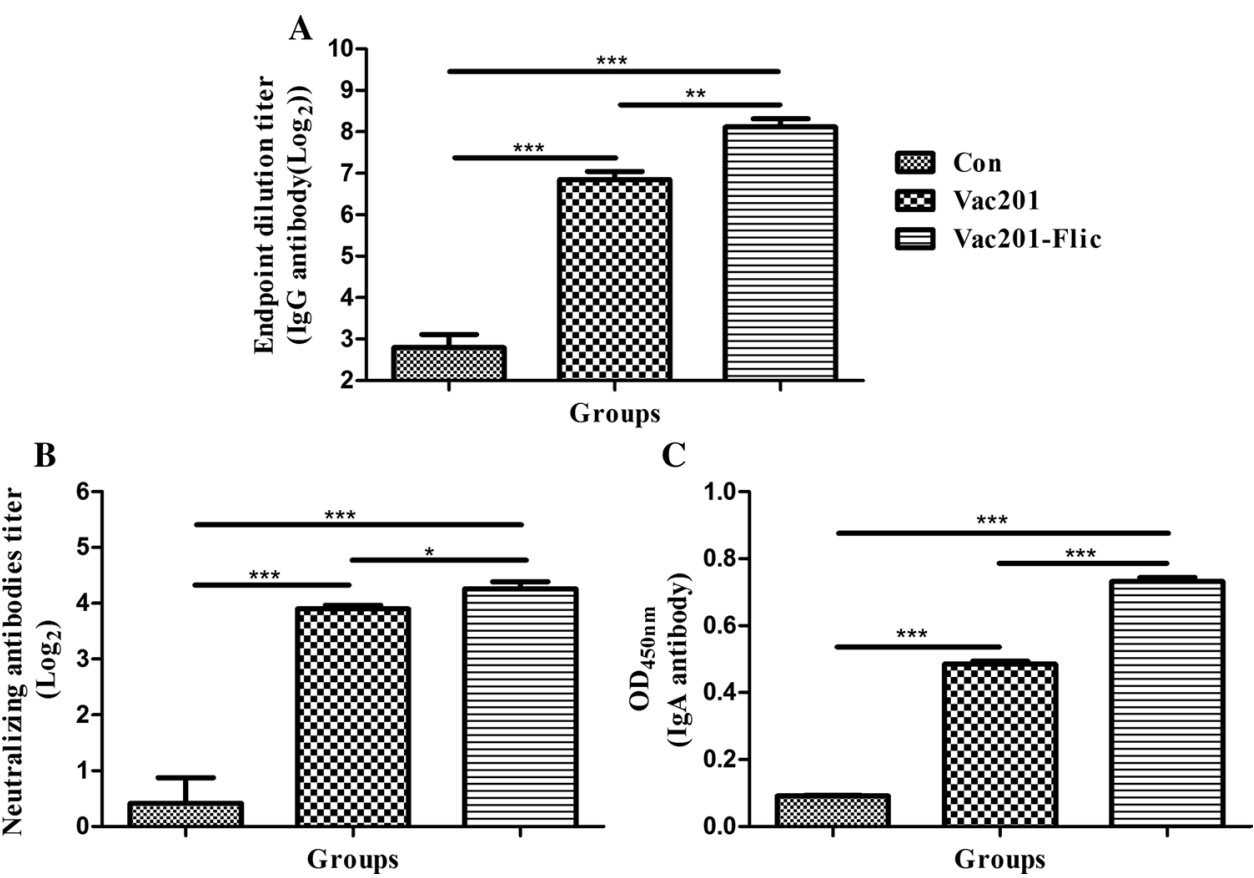

Groups 
with immune protection against PEDV [26], and FliC was demonstrated to induce a mucosal immune response, PEDV-specific IgA levels in the colostrum samples were determined by AniGen PED IgA Ab ELISA. The optical densities $\left(\mathrm{OD}_{450}\right)$ of the colostrum samples from the pregnant sows that were vaccinated intranasally with Vac201FliC $(1.153 \pm 0.044)$ were significantly higher than those of animals immunized with PBS alone $(0.082 \pm 0.009)$ or Vac201 $(0.583 \pm 0.030)$ (both $P<0.001)$ (Fig. 4C).

\section{Cellular immune responses in pregnant sows}

We also assessed the cellular immune responses in pregnant sows vaccinated with Vac201-FliC, Vac201 or PBS. As shown in Fig. 5A, the highest SI $(3.243 \pm 0.060)$ was observed in the pregnant sows inoculated with Vac201FliC, and this differed significantly from the SIs of animals inoculated with PBS $(0.983 \pm 0.012)$ or Vac201 $(1.927 \pm 0.039)$. Similar trends were also observed for IFN- $\gamma$ expression and IFN- $\gamma$ mRNA assays, which are shown in Fig. 5B and C, respectively.

\section{Antibody responses in 5-day-old piglets}

The antibody responses of 5-day-old piglets (fifteen piglets in each group) prior to challenge are presented in Fig. 6. Piglets delivered from sows inoculated with Vac201-FliC had higher levels of PEDV-N-protein-specific antibodies (1:277.3) than those inoculated with PBS (1:6.9) or Vac201 (1:115.2). Piglets born to Vac201-FliC-vaccinated mothers had significantly more neutralizing antibodies than piglets from sows inoculated with PBS or Vac201 $(P<0.001$ and $P<0.05$, respectively). As shown in Fig. $6 \mathrm{C}$, the $\mathrm{OD}_{450}$ values of the serum samples ( $\operatorname{Ig} \mathrm{A}$ antibody) from piglets delivered from sows that were inoculated intranasally with Vac201-FliC $(0.732 \pm 0.011)$ were significantly higher than those of piglets delivered from the sows that were inoculated with PBS $(0.091 \pm 0.001)$ or Vac201 $(0.485 \pm 0.009)$ (both $P<0.001)$.

\section{Clinical signs in piglets after PEDV challenge}

After challenge with PEDV strain AH2012/12, the piglets were monitored for clinical signs. The piglets born to sows vaccinated with PBS displayed lethargy and diarrheic feces at $6 \mathrm{~h}$ post-challenge (hpc), and thereafter exhibited

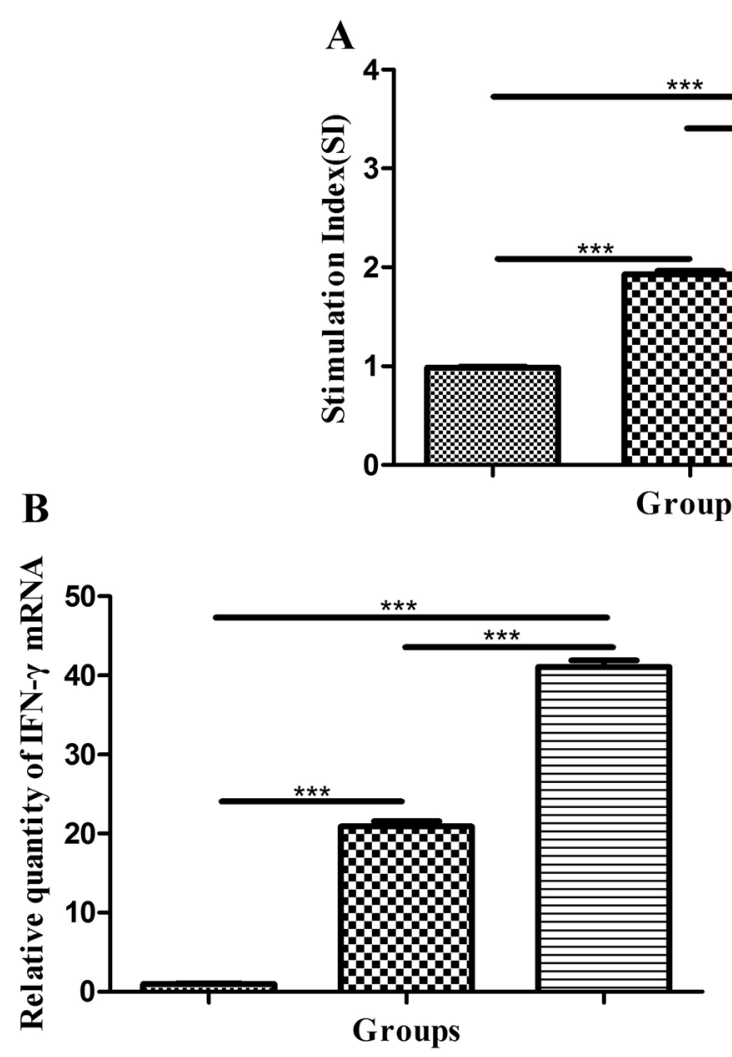

Fig. 5 Cellular immune responses in pregnant sows inoculated at farrowing with PBS, Vac201, or Vac201-FliC. PBMCs were isolated to assess lymphocyte proliferation (A), IFN- $\gamma$ mRNA levels (B), and 

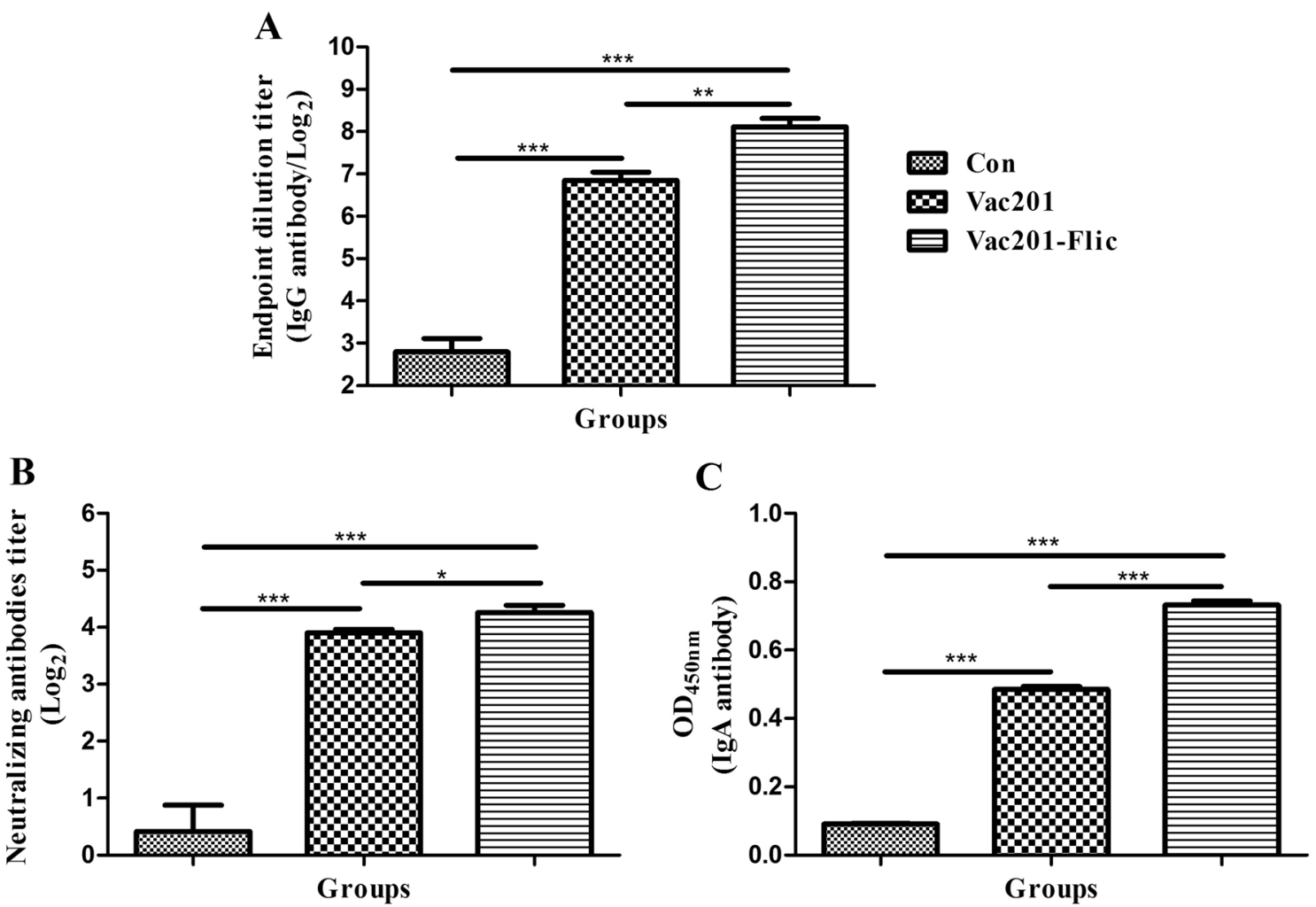

Fig. 6 Antibody responses in 5-day-old piglets (15 piglets in each group) from sows immunized with different inactivated vaccine formulations. Serum samples were collected from 5-day-old piglets before challenge with PEDV strain AH2012/12. PEDV-specific anti-

body levels (OD values) (A) and neutralizing antibody levels (B) were determined as described in Materials and methods. Data presented are the mean \pm SEM of 15 piglets per group

depression, severe watery diarrhea with vomiting and severe dehydration. In contrast, piglets delivered from the Vac201FliC-inoculated sows displayed normal appetite and feces and a lower score for fecal density than those in the other groups (Fig. 7A).

An RT-qPCR analysis of fecal samples showed that the variations in viral shedding in the different groups showed a similar trend. The level of viral shedding was significantly lower in piglets born to the Vac201-FliC-inoculated sows at all sampling times when compared with those from the control group $(P<0.05$ or $P<0.01)$. Similar levels of viral shedding were observed in piglets born to sows immunized with Vac201-FliC or Vac201 only at $6 \mathrm{hpc}$. At all other sampling times, viral shedding differed significantly between the piglets born to these two groups of sows $(P<0.05)$ (Fig. 7B).

The mortality of piglets after challenge with PEDV strain AH2012/12 was $13.33 \%$ in the Vac201-FliC-vaccinated group (2/15), compared with $86.67 \%$ in the PBS-vaccinated group (13/15) and 46.67\% (7/15) in the Vac201-vaccinated group (Fig. 7C). To illustrate the degrees of diarrhea in the three different immunized groups, we took pictures of the piglets with diarrhea, which were more visually apparent than using a scoring system to assess diarrheal severity

(Fig. 8). Histopathological examination of the intestinal mucosa of piglets from PBS-vaccinated sows revealed severe atrophy, damaged villi, and epithelial cell shedding. Damaged villi and condensation of the lamina propria were seen in the intestines of piglets from the Vac201-vaccinated group. Very few histopathological changes were observed in the Vac201-FliC piglets than in the other ones (Fig. 8).

\section{Discussion}

Since late 2010, PED caused by a variant PEDV has been prevalent in the swine industry in China, despite most swine populations having been immunized with commercial vaccines derived from PEDV strain CV 777 [7, 8, 13, 27]. Four- to 13-day-old piglets are predominantly protected from PEDV infection by specific $\operatorname{IgG}$ and $\operatorname{Ig} A$ antibodies in the colostrum and milk of immune sows, and the duration of immunity depends on the antibody titers of the mother [25]. Therefore, inducing high titers of PEDV-specific antibodies directed against the prevalent PEDV strain is one of the main aims in the development of PEDV vaccines. Considering the high degree of amino acid sequence similarity of 


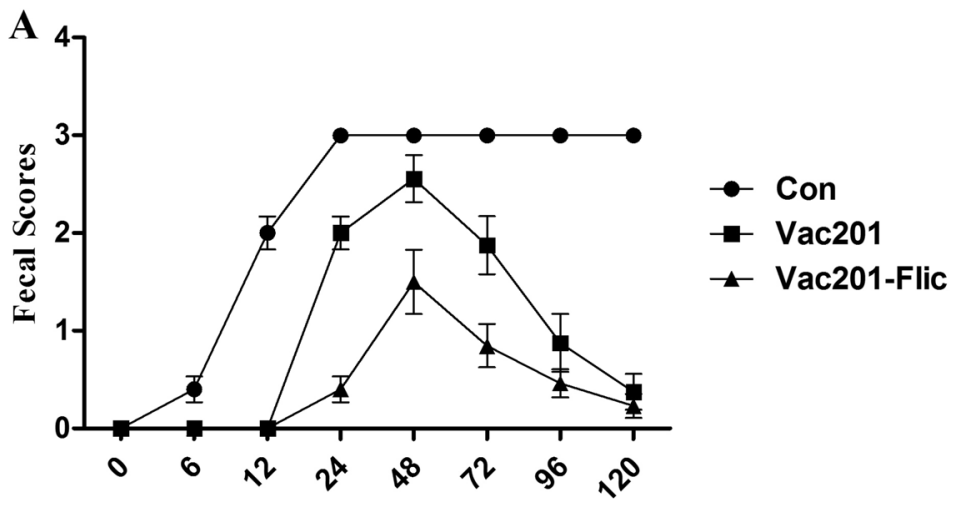

Hours post-challenge

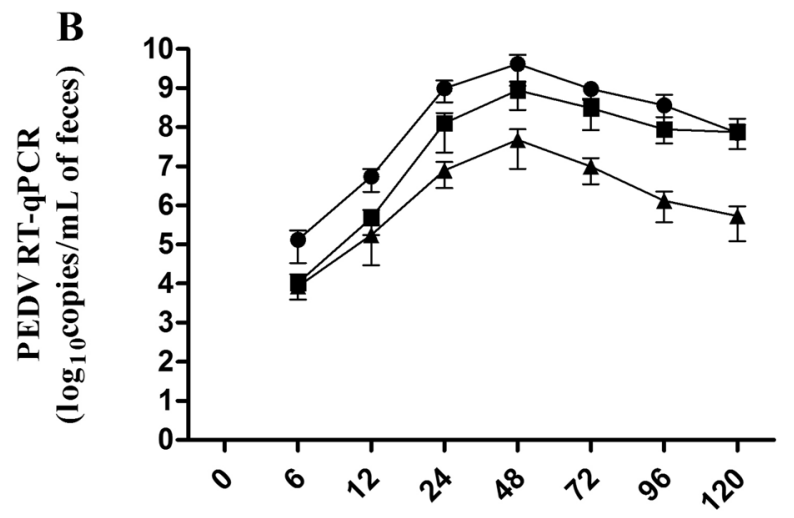

Hours post-challenge

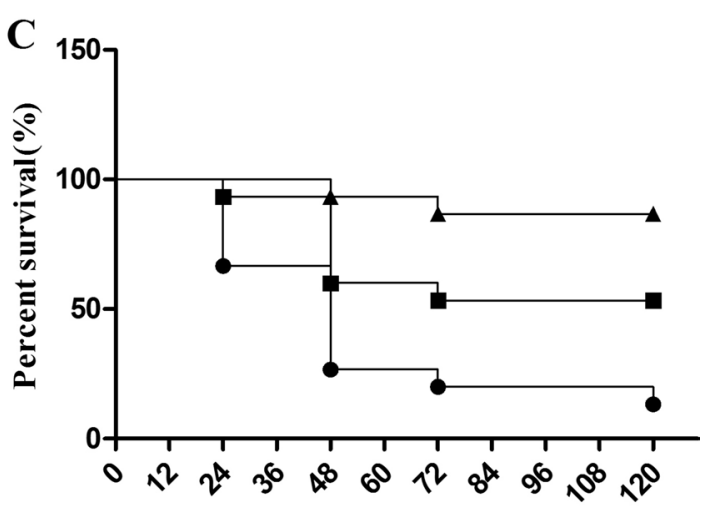

Hours post-challenge

Fig. 7 Clinical signs of PED in piglets after challenge with PEDV strain AH2012/12. (A) Fecal scores. (B) RT-PCR detection of viral shedding. (C) piglet mortality
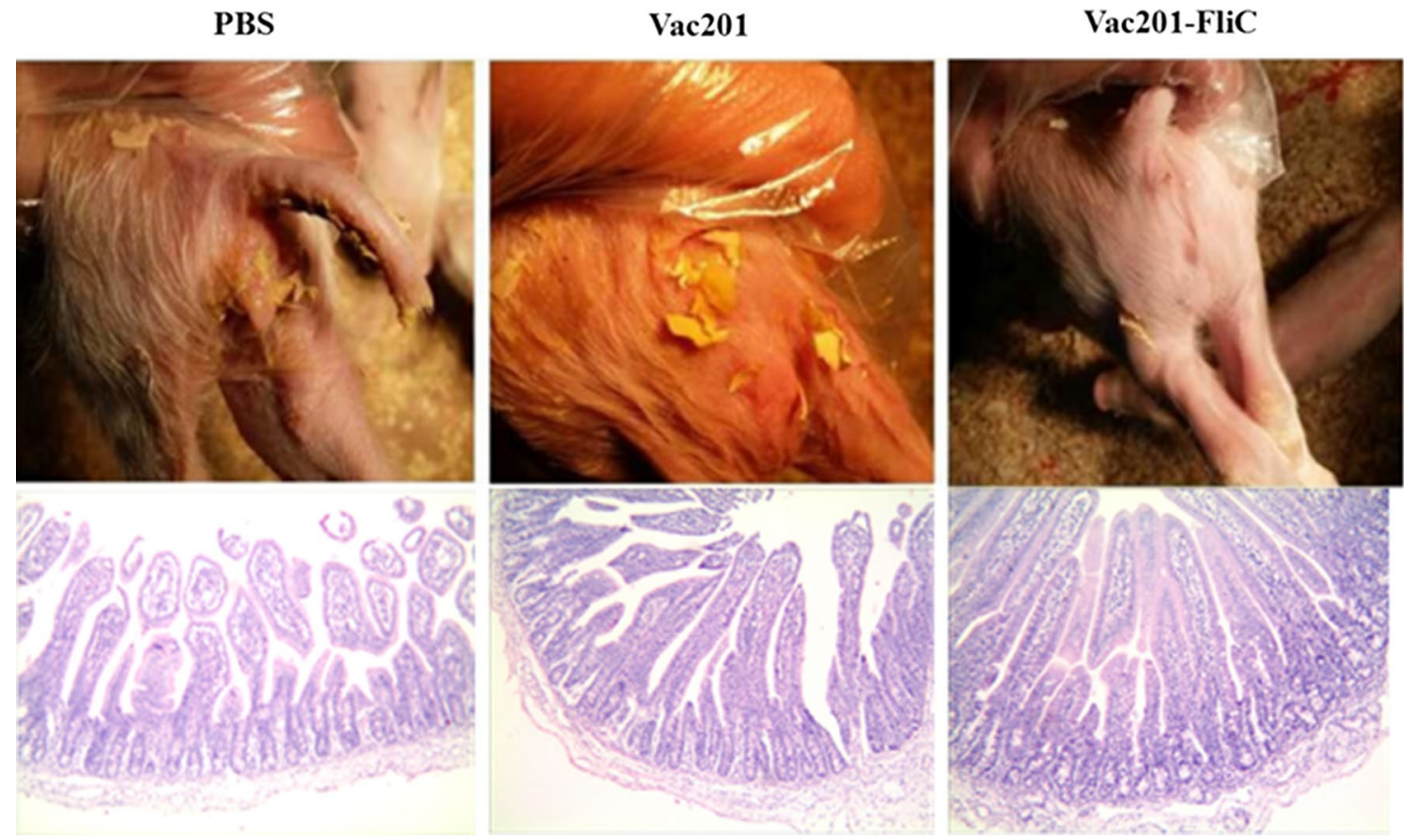

Fig. 8 Macroscopic examination of feces from piglets of sows immunized with different inactivated vaccine formulations 
the $\mathrm{N}$ proteins among different PEDV strains and the high immunogenicity of this protein, and its ability to induce both $\mathrm{T}$ cell and B cell immune responses, PEDV-N-proteinspecific antibody titers were evaluated in our study to assess the immune responses of immunized animals.

Currently, research in the field of PEDV vaccine development mainly focuses on screening new PEDV strains or optimizing the dose of PEDV antigens [2, 6, 20, 21, 24]. In this study, we attempted to identify a PEDV vaccine adjuvant that could efficiently induce N-protein-specific IgG immune responses, mucosal immune responses in pregnant sows, ensuring delivery of colostral IgA antibody to piglets in order to protect them from PEDV infection, as well as T cell responses.

Flagellin, a ligand of TLR5, efficiently triggers efficient mixed Th1 and Th2 cell responses, as well as IgA mucosal immunity [4]. Numerous studies have demonstrated that FliC contributes to the efficacy of various novel vaccines against Yersinia pestis [15], West Nile virus [23], Plasmodium falciparum [3], tetanus toxoid [18], and influenza virus [1,5]. Studies have demonstrated that a flagellin-adjuvanted PED subunit vaccine elicits enhanced systemic, local mucosal and $\mathrm{T}$ cell immune responses in mice and pigs [20,21]. However, there have been no studies of FliC as a mucosal adjuvant for inactivated PEDV vaccines in pregnant sows for which the immune responses in newborn piglets were evaluated after delivery.

In this study, the dose of PEDV vaccine was equivalent to approximately $10^{6} \mathrm{TCID}_{50}$ of inactivated virus, which was similar to that of most commercially inactivated vaccines. Furthermore, compared with PEDV subunit vaccines, inactivated vaccines are more cost-efficient and more simply prepared. Thus, we aimed to develop an efficient inactivated PEDV vaccine in response to the serious economic consequences of PEDV on swine farms in China.

After immunization, no adverse side effects (such as vomiting or diarrhea) were observed in the experimental pregnant sows, which indicated the safety of the Vac201FliC vaccine. We prepared the inactivated PEDV vaccine based on previous experience, using this information to determine the dose of inactivated PEDV, the dose of adjuvant Montanide ${ }^{\mathrm{TM}}$ ISA201, and the amount of flagellin. In future studies, we will determine what ratio of virus, adjuvant and FliC is necessary for an optimal immune response in the sow and passive transfer of $\operatorname{Ig} \mathrm{A}$ antibodies to the piglets. In this study, we used PEDV and FliC separately rather than as a fusion protein of the target antigen and FliC [20, 21]. This method was used to enable separate modification of PEDV while preserving the antigenicity of the virus. In this study, we only evaluated the efficacy of the vaccines through the intranasal route and not the intramuscular route. Because FliC is a mucosal adjuvant, we hypothesized that nasal immunization could efficiently induce a mucosal immune response, which could provide better mucosal immunity against PEDV infection. In the future, we will conduct more studies using different immunization routes and ratios of PEDV vaccine components to clarify the role of FliC.

Our results demonstrated that Vac201-FliC not only increased the production of PEDV-N-protein-specific IgG antibodies and increased the neutralizing antibody titers in serum and colostrum samples, but also enhanced the levels of PEDV-specific IgA antibodies in colostrum samples. We found that the titers of serum and colostral antibodies from pregnant sows immunized with PBS might be related to the background level of detection in the assays. However, the significant differences in antibody titers between the viral immunization group and the control group indicated the success of the experiment. Meanwhile, the significantly higher levels of PEDV-specific IgA antibodies in the colostrum partially explains the enhanced levels of PEDV-specific IgA antibodies in serum of piglets delivered from Vac201-FliC-immunized sows. Sows immunized with Vac201-FliC showed not only enhanced humoral immune responses but also improved cellular immunity compared with those in the groups immunized with PBS or Vac201. The significantly more potent immune responses induced by Vac201-FliC resulted in a mortality rate as low as $13.33 \%$ after challenge with PEDV strain AH2012/12. The results of this study indicate that Vac201-FliC is a promising candidate vaccine for protecting piglets against PEDV infection.

To our knowledge, this is the first report of an inactivated PEDV vaccine adjuvanted with FliC in pregnant sows. Flagellin, a mucosal adjuvant that is compatible with inactivated or killed PEDV vaccines, enhanced production of serum IgG and IgA antibodies and colostrum IgA antibodies by a PEDV vaccine administrated intranasally. The vaccine conferred protective immunity on piglets against PEDV infection.

Acknowledgements This work was supported by the the National Natural Sciences Foundation of China (31872481, 31802167 and 31802220), the Jiangsu Province Natural Sciences Foundation (BK20190003), Jiangsu Agricultural Science and Technology Innovation Fund (CX (19) 2020), the Open Project Fund of Jiangsu Provincial Key Construction Laboratory of Probiotics Preparation (JSYSZJ2017004) and Six Talent Peaks in Jiangsu Province (NY-045). It was supported by SEPPIC, Inc. (China, Shanghai). We thank Liwen Bianji, Edanz Editing China (www.liwenbianji.cn/ac), for editing the English text of a draft of this manuscript.

Author contributions $\mathrm{XX}, \mathrm{LD}, \mathrm{BL}$ and $\mathrm{KH}$ designed the experiment. Sampling of porcine serum, faeces and intestines were mainly performed by LD, BS, XX, ZY, JZ and RG. LD and BF analyzed the results with guidance from $B L . X X, L D$ and $B L$ wrote the main manuscript text. All authors took part in discussion and interpretation of results. All authors read, advised and approved the final manuscript. 


\section{Compliance with ethical standards}

Conflict of interest None of the authors has a commercial or other association that might pose a conflict of interest.

\section{Reference}

1. Adar Y, Singer Y, Levi R, Tzehoval E, Perk S, Banet-Noach C, Nagar S, Arnon R, Ben-Yedidia T (2009) A universal epitopebased influenza vaccine and its efficacy against H5N1. Vaccine 27:2099-2107

2. Annamalai T, Lin CM, Gao X, Liu X, Lu Z, Saif LJ, Wang Q (2017) Cross protective immune responses in nursing piglets infected with a US spike-insertion deletion porcine epidemic diarrhea virus strain and challenged with an original US PEDV strain. Vet Res 48:61

3. Bargieri DY, Rosa DS, Braga CJ, Carvalho BO, Costa FT, Espindola NM, Vaz AJ, Soares IS, Ferreira LC, Rodrigues MM (2008) New malaria vaccine candidates based on the Plasmodium vivax merozoite surface protein-1 and the TLR-5 agonist Salmonella Typhimurium FliC flagellin. Vaccine 26:6132-6142

4. Bates JT, Honko AN, Graff AH, Kock ND, Mizel SB (2008) Mucosal adjuvant activity of flagellin in aged mice. Mech Ageing Dev 129:271-281

5. Ben-Yedidia T, Arnon R (2007) Epitope-based vaccine against influenza. Expert Rev Vaccines 6:939-948

6. Chang YC, Kao CF, Chang CY, Jeng CR, Tsai PS, Pang VF, Chiou HY, Peng JY, Cheng IC, Chang HW (2017) Evaluation and comparison of the pathogenicity and host immune responses induced by a G2b Taiwan porcine epidemic diarrhea virus (Strain Pintung 52 ) and its highly cell-culture passaged strain in conventional 5-week-old pigs. Viruses 9:121-1-121-15

7. Chen F, Pan Y, Zhang X, Tian X, Wang D, Zhou Q, Song Y, Bi Y (2012) Complete genome sequence of a variant porcine epidemic diarrhea virus strain isolated in China. J Virol 86:12448

8. Chen J, Liu X, Shi D, Shi H, Zhang X, Feng L (2012) Complete genome sequence of a porcine epidemic diarrhea virus variant. J Virol 86:3408

9. Chen Q, Li G, Stasko J, Thomas JT, Stensland WR, Pillatzki AE, Gauger PC, Schwartz KJ, Madson D, Yoon KJ, Stevenson GW, Burrough ER, Harmon KM, Main RG, Zhang J (2014) Isolation and characterization of porcine epidemic diarrhea viruses associated with the 2013 disease outbreak among swine in the United States. J Clin Microbiol 52:234-243

10. Du L, Li B, Pang F, Yu Z, Xu X, Fan B, Tan Y, He K, Huang K (2017) Porcine GPX1 enhances GP5-based DNA vaccination against porcine reproductive and respiratory syndrome virus. Vet Immunol Immunopathol 183:31-39

11. Du L, Pang F, Yu Z, Xu X, Fan B, Huang K, He K, Li B (2017) Assessment of the efficacy of two novel DNA vaccine formulations against highly pathogenic porcine reproductive and respiratory syndrome virus. Sci Rep 7:41886

12. Fan B, Yu Z, Pang F, Xu X, Zhang B, Guo R, He K, Li B (2017) Characterization of a pathogenic full-length cDNA clone of a virulent porcine epidemic diarrhea virus strain AH2012/12 in China. Virology 500:50-61

13. Gao Y, Kou Q, Ge X, Zhou L, Guo X, Yang H (2013) Phylogenetic analysis of porcine epidemic diarrhea virus field strains prevailing recently in China. Arch Virol 158:711-715
14. Honko AN, Mizel SB (2004) Mucosal administration of flagellin induces innate immunity in the mouse lung. Infect Immun 72:6676-6679

15. Honko AN, Sriranganathan N, Lees CJ, Mizel SB (2006) Flagellin is an effective adjuvant for immunization against lethal respiratory challenge with Yersinia pestis. Infect Immun 74:1113-1120

16. Huleatt JW, Jacobs AR, Tang J, Desai P, Kopp EB, Huang Y, Song L, Nakaar V, Powell TJ (2007) Vaccination with recombinant fusion proteins incorporating Toll-like receptor ligands induces rapid cellular and humoral immunity. Vaccine 25:763-775

17. Lee C (2015) Porcine epidemic diarrhea virus: an emerging and re-emerging epizootic swine virus. Virol J 12:193

18. Lee SE, Kim SY, Jeong BC, Kim YR, Bae SJ, Ahn OS, Lee JJ, Song HC, Kim JM, Choy HE, Chung SS, Kweon MN, Rhee JH (2006) A bacterial flagellin, Vibrio vulnificus FlaB, has a strong mucosal adjuvant activity to induce protective immunity. Infect Immun 74:694-702

19. Li B, Du L, Yu Z, Sun B, Xu X, Fan B, Guo R, Yuan W, He K (2017) Poly (d, L-lactide-co-glycolide) nanoparticle-entrapped vaccine induces a protective immune response against porcine epidemic diarrhea virus infection in piglets. Vaccine 35:7010-7017

20. Li Q, Peng O, Wu T, Xu Z, Huang L, Zhang Y, Xue C, Wen Z, Zhou Q, Cao Y (2018) PED subunit vaccine based on COE domain replacement of flagellin domain D3 improved specific humoral and mucosal immunity in mice. Vaccine 36:1381-1388

21. Li Q, Xu Z, Wu T, Peng O, Huang L, Zhang Y, Xue C, Wen Z, Zhou Q, Cao Y (2018) A flagellin-adjuvanted PED subunit vaccine improved protective efficiency against PEDV variant challenge in pigs. Vaccine 36:4228-4235

22. Li W, Li H, Liu Y, Pan Y, Deng F, Song Y, Tang X, He Q (2012) New variants of porcine epidemic diarrhea virus, China, 2011. Emerg Infect Dis 18:1350-1353

23. McDonald WF, Huleatt JW, Foellmer HG, Hewitt D, Tang J, Desai P, Price A, Jacobs A, Takahashi VN, Huang Y, Nakaar V, Alexopoulou L, Fikrig E, Powell TJ (2007) A West Nile virus recombinant protein vaccine that coactivates innate and adaptive immunity. J Infect Dis 195:1607-1617

24. Opriessnig T, Gerber PF, Shen H, de Castro A, Zhang J, Chen Q, Halbur P (2017) Evaluation of the efficacy of a commercial inactivated genogroup $2 b$-based porcine epidemic diarrhea virus (PEDV) vaccine and experimental live genogroup $1 \mathrm{~b}$ exposure against $2 \mathrm{~b}$ challenge. Vet Res 48:69

25. Paudel S, Park JE, Jang H, Hyun BH, Yang DG, Shin HJ (2014) Evaluation of antibody response of killed and live vaccines against porcine epidemic diarrhea virus in a field study. Vet Q 34:194-200

26. Song DS, Oh JS, Kang BK, Yang JS, Moon HJ, Yoo HS, Jang YS, Park BK (2007) Oral efficacy of Vero cell attenuated porcine epidemic diarrhea virus DR13 strain. Res Vet Sci 82:134-140

27. Sun RQ, Cai RJ, Chen YQ, Liang PS, Chen DK, Song CX (2012) Outbreak of porcine epidemic diarrhea in suckling piglets, China. Emerg Infect Dis 18:161-163

28. Wang D, Fang L, Xiao S (2016) Porcine epidemic diarrhea in China. Virus Res 226:7-13

29. Zhang X, Pan Y, Wang D, Tian X, Song Y, Cao Y (2015) Identification and pathogenicity of a variant porcine epidemic diarrhea virus field strain with reduced virulence. Virol J 12:88

Publisher's Note Springer Nature remains neutral with regard to jurisdictional claims in published maps and institutional affiliations. 